TIPA. Travaux interdisciplinaires sur la parole et le langage

27 | 2008

Apprentissage et acquisition d'une langue seconde

\title{
Éditorial
}

\section{Martine Faraco}

\section{(2) OpenEdition}

Journals

Édition électronique

URL : https://journals.openedition.org/tipa/247

DOI : 10.4000/tipa. 247

ISSN : 2264-7082

Éditeur

Laboratoire Parole et Langage

Édition imprimée

Date de publication : 1 juin 2008

ISSN : 1621-0360

\section{Référence électronique}

Martine Faraco, «Éditorial », TIPA. Travaux interdisciplinaires sur la parole et le langage [En ligne], 27 |

2008, mis en ligne le 04 décembre 2012, consulté le 08 septembre 2021. URL : http://

journals.openedition.org/tipa/247 ; DOI : https://doi.org/10.4000/tipa.247

Ce document a été généré automatiquement le 8 septembre 2021.

\section{(c) $(1)(9)$}

La revue TIPA. Travaux interdisciplinaires sur la parole et le langage est mise à disposition selon les termes de la licence Creative Commons Attribution - Pas d'Utilisation Commerciale - Pas de Modification 4.0 International. 


\section{Éditorial}

\section{Martine Faraco}

1 Que l'on soit praticien de la classe de langue, didacticien, psycholinguiste ou phonéticien, ce numéro thématique sur l'apprentissage et l'acquisition d'une langue nouvelle ne laissera pas indifférent. Les pistes seront nombreuses, contradictoires, ressemblantes, identiques ou différentes, toujours est-il que la même question poursuit les chercheurs : comment apprend-on et acquiert-on une langue maternelle et une langue seconde ? De la même façon ou de façon différente?

2 Il suffira de pénétrer dans l'atelier d'écriture que Brigitte Bonnefoy anime pour des étudiants étrangers apprenant le français, pour saisir toute la subtilité des rapports qu'entretiennent la langue maternelle et la langue seconde. Ainsi, luttant avec une écriture coupée de la norme de sa langue seconde, le scripteur se focalisera non pas sur le secondaire (le thème de son écrit), mais sur l'essentiel, la transmission de soi à l'autre par l'écriture.

3 Évidemment, pour écrire, il faut connaître le système grammatical de la L2. C'est justement sur ce thème que s'interrogent Haydee Carrasco et Cheryl Frenck-Mestre en examinant, en amont de la production, la représentation de la grammaire en langue seconde. Elles font ainsi un état des lieux des recherches portant sur la façon dont la grammaire de la langue maternelle et de la langue seconde cohabitent, se complètent, ou encore se parasitent, en fonction de l'éloignement ou de la proximité des deux langues ou en fonction d'une série d'autres facteurs.

4 Quant à la perception la parole, Sophie Dufour et Noël Nguyen prennent comme point de départ l'influence de la langue maternelle. En effet, pour ces chercheurs, il semble clair que l'apprentissage d'une langue maternelle, dans les premières années de vie, affecte l'auditeur d'une écoute sélective le rendant sourd à des contrastes phonologiques de la langue cible, non discriminatoires dans la langue source, voire à une variété régionale à l'intérieur de cette dernière.

5 Les variétés régionales, ou plus exactement les accents régionaux de l'anglais, sont d'ailleurs l'objet de la recherche de Kizzi Edensor qui note que la non-familiarité avec tel ou tel accent régional ralentit la compréhension chez un non-natif. 
6 C'est par le biais de l'intonation que Martine Faraco et Christian Cavé appréhendent la compréhension d'une L2. Ils montrent que le degré de complexité syntaxique et/ou lexicale peut interférer sur la perception de l'intonation. En revanche, curieusement, l'effet du locuteur est secondaire pour les non-natifs par rapport aux natifs.

7 Nadine Herry-Bénit, quant à elle, revient à des préoccupations plus didactiques par son expérience mettant en contraste, dans des classes d'anglais, la méthode silent way et une méthode traditionnelle.

8 Hors de la classe, cette fois, Aïno Niklas-Salminen s'intéresse à l'acquisition du lexique par une enfant bilingue finnois et français. L'analyse des énoncés hybrides donne les premières pistes de recherche pour découvrir la nature des mélanges lexicaux, dans une pratique bilingue authentique.

9 Enfin, le corpus ANGLISH, présenté par Anne Tortel et permettant de travailler sur des enregistrements en anglais britannique L1 et L2, prouve, si besoin était, que les communautés enseignante et scientifique tendent à se doter d'outils communs pour qu'avance notre connaissance de l'apprentissage et de l'acquisition d'une langue seconde.

Bonne lecture!

\section{AUTEUR}

\section{MARTINE FARACO}

Laboratoire Parole et Langage 